\title{
HILBERT SPACE IS HOMEOMORPHIC TO THE COUNTABLE INFINITE PRODUCT OF LINES ${ }^{1}$
}

\author{
BY R. D. ANDERSON ${ }^{2}$ \\ Communicated by V. Klee, January 24, 1966
}

1. Introduction. In this paper, Hilbert space, denoted by $l_{2}$, is understood to be the space of all sequences $\left(x_{i}\right)$ such that $\sum_{i=1}^{\infty} x_{i}^{2}<\infty$ with $d\left(\left(x_{i}\right),\left(x_{i}^{\prime}\right)\right)=\left(\sum_{i=1}^{\infty}\left(x_{i}-x_{i}^{\prime}\right)^{2}\right)^{1 / 2}$. We let the countable infinite product of lines be regarded as $s=\prod_{i=1}^{\infty} I_{i}^{0}$ where, for each $i>0, I_{i}^{0}$ denotes the open interval $(0,1)$.

Let the symbol " " mean "is homeomorphic to." We shall prove

THEOREM I. $l_{2} \sim s$.

As a consequence of this theorem it is possible to investigate topological properties of $l_{2}$ as topological properties of $s$. In turn $s$ is a "natural" subset of the Hilbert cube (the countable infinite product of closed intervals) which facilitates the study of $s$.

In 1928 in [5, pp. 94-96] Fréchet raised the general question as to which linear topological spaces were homeomorphic to each other. Specifically he asked whether $l_{2}$ (called $\Omega$ ) was homeomorphic to $s$ (called $E_{\omega}$ ).

In 1932 in [2, p. 233], Banach stated that Mazur had shown that $s$ was not homeomorphic to $l_{2}$. Subsequently it was understood that the question was still open.

The topological classification of complete linear metric spaces initiated by Fréchet has been the subject of considerable research activity with noteworthy contributions by Bessaga, Kadec, Klee and Pelczyński among others. See the bibliography in [3]. Particular attention has been given to Fréchet spaces: locally convex complete linear metric spaces. With Theorem I of this paper and recent profound results of Kadec and of Bessaga and Pełczyński, the topological classification of separable infinite-dimensional Fréchet spaces is now complete. All such spaces are homeomorphic to each other.

The results leading to this theorem are the following. In a paper to be published in Dokl. Akad. Nauk SSSR, Kadec gives a proof of the theorem "All separable infinite-dimensional Banach Spaces are homeomorphic." Earlier in [4] and in [3, Theorem 9.2], Bessaga and Pełczyński have shown "Under the conjecture that all separable in-

1 This research was supported under NSF Grant GP 4893.

2 The author is indebted to A. Lelek and A. Pełczynski for interesting conversations associated with this problem. 
finite-dimensional Banach spaces are homeomorphic with $l_{2}$, every separable infinite-dimensional Fréchet space $X$, with $X \neq s$, is homeomorphic with $l_{2}$." Therefore, with Theorem I of this paper, the classification is complete.

2. The strategy of the proof of Theorem I. The proof of Theorem I uses only standard topological methods and two recent results, (A) and (B) below, which are not proved here. (However, an outline of the proof of (B) is given in §3.) Otherwise, this paper is self-contained.

(A) (Bessaga-Pełczyński) [3] or [4].

$$
l_{2} \sim l_{2} \times s \text {. }
$$

(B) [1]. For any separable metric space $Z$ and any countable collection $\left\{K_{i}\right\}_{i \gtrless 1}$ of compact subsets of $Z \times s$,

$$
\left[(Z \times s) \backslash \bigcup_{i=1}^{\infty} K_{i}\right] \sim Z \times s
$$

Taking $Z$ as a single-point set, the following theorem is a corollary of (B).

(C) For any countable collection $\left\{K_{i}\right\}_{i \geqq 1}$ of compact subsets of $s$,

$$
\left[s \backslash \bigcup_{i=1}^{\infty} K_{i}\right] \sim s .
$$

Taking $Z \sim l_{2}$, the following theorem is a corollary of (A) and (B). (D) For any countable collection $\left\{K_{i}\right\}_{i \geq 1}$ of compact subsets of $l_{2}$,

$$
\left[l_{2} \backslash \bigcup_{i=1}^{\infty} K_{i}\right] \sim l_{2}
$$

With these observations, the strategy of the proof is the following. We exhibit, in $\$ 4$, a particular set $l_{2}$ which, by (D), we show to be homeomorphic to $l_{2}$. Then, in $\$ 5$, we show (E) a homeomorphism $f$ of $\tilde{l}_{2}$ into $s$ such that $s \backslash f\left(l_{2}\right)$ is the countable union of compact sets. By $(\mathrm{C}), f\left(\mathcal{l}_{2}\right) \sim s$. Thus we have

$$
l_{2} \stackrel{\mathrm{D}}{\sim} \tilde{l}_{2} \stackrel{\mathrm{E}}{\sim} f\left(\tilde{l}_{2}\right) \stackrel{\mathrm{C}}{\sim}
$$

and hence $l_{2} \sim s$.

3. Outline of a proof of (B). We have $s=\prod_{j=1}^{\infty} I_{j}^{0}$ where, for each $j, I_{j}^{0}$ is the open interval $(0,1)$. Then we may write $s$ as $\pi_{i=1}^{\infty} s_{i}$ where, for each $i, s_{i}$ is a countable infinite product of factors $I_{j}^{0}$. Regarding $Z \times s$ as $Z \times \pi_{i=1}^{\infty} s_{i}$, we let $g_{i}$ be the projection of $Z \times s$ onto $s_{i}$. Observe 
that, for each $i$ with $K_{i}$ as in the statement of (B), $g_{i}\left(K_{i}\right)$ is compact.

It can be shown (as in $\$ 3$ of [1]) that, for each $i$, there is a homeomorphism $\rho_{i}$ of $s_{i}$ onto itself and a factorization $s_{i}=s_{i}^{\prime} \times s_{i}^{\prime \prime}$ (where each of $s_{i}^{\prime}$ and $s_{i}^{\prime \prime}$ is a countable infinite product of factors $I_{j}^{0}$ ) such that if $\sigma_{i}$ denotes the projection of $s_{i}^{\prime} \times s_{i}^{\prime \prime}$ onto $s_{i}^{\prime}$, then $\sigma_{i} \rho_{i} g_{i}\left(K_{i}\right)$ is a single point. In other words, $\rho_{i} g_{i}\left(K_{i}\right)$ has infinite deficiency in $s_{i}$.

Let $\rho$ be the homeomorphism of $Z \times \prod_{i=1}^{\infty} s_{i}$ onto itself defined coordinatewise as $\rho_{i}$ on $s_{i}$ and as the identity on $Z$. It now suffices to exhibit a homeomorphism of $(Z \times s) \backslash \bigcup_{i=1}^{\infty} \rho\left(K_{i}\right)$ onto $Z \times s$.

In what follows we let $K_{0}=\rho\left(K_{0}\right)$ be the null set.

Considering $Z \times s$ as $Z \times \prod_{i=1}^{\infty}\left(s_{i}^{\prime} \times s_{i}^{\prime \prime}\right)$, we may exhibit (as in $\S 5$ of [1]) a sequence $\left\{h_{i}\right\}_{i \geqq 1}$ such that

(1) for each $i \geqq 1, h_{i}$ is a homeomorphism of $(Z \times s) \backslash \bigcup_{j=0}^{i} \rho\left(K_{j}\right)$ onto $(Z \times s) \backslash \bigcup_{j=0}^{i-1} \rho\left(K_{j}\right)$,

(2) for each $i \geqq 1, h_{i}$ affects only coordinates in $s_{i}^{\prime}$, and

(3) the infinite composition $\cdots \cdot h_{3} \cdot h_{2} \cdot h_{1}$ is a homeomorphism $h$ carrying $(Z \times s) \backslash \bigcup_{j=0}^{\infty} \rho\left(K_{j}\right)$ onto $Z \times s$.

Thus $h \rho$ will be the desired homeomorphism of $(Z \times s) \backslash \bigcup_{j=0}^{\infty} K_{j}$ onto $Z \times s$.

In conclusion, we remark that the homeomorphism $h_{i}$ can be considered as a modification of a homeomorphism defined coordinatewise as (1) the identity on coordinate spaces other than $s_{i}^{\prime}$ and (2) a homeomorphism which moves the single point $\sigma_{i} g_{i} \rho\left(K_{i}\right)$ off $s_{i}^{\prime}$. The modification requires that $h_{i}$ move only $\rho\left(K_{i}\right) \backslash \bigcup_{j=0}^{i-1} \rho\left(K_{j}\right)$ off $Z \times s$ rather than the larger set $g_{i}^{-1} \sigma_{i}^{-1}\left(\sigma_{i} g_{i} \rho\left(K_{i}\right)\right) \backslash \bigcup_{j=0}^{i-1} \rho\left(K_{j}\right)$.

4. Description of $l_{2}$. For each $i \geqq 1$, let $W_{i}=\left\{\left(x_{j}\right) \mid\left(x_{j}\right) \in l_{2}\right.$ and for all $\left.k>i, x_{k}=0\right\}$. Then for each $i, W_{i}$ is homeomorphic to the Euclidean space $E_{i}$ and is the countable union of compact sets. Thus $W=\cup_{i=1}^{\infty} W_{i}$ is the countable union of the compact sets. Let $M_{1}$ $=\left\{\left(x_{j}\right) \mid\left(x_{j}\right) \in l_{2} \backslash W\right.$ and $\left.x_{1}=1\right\}$. Let $\tilde{l}_{2}=\left\{\left(x_{j}\right) \mid\left(x_{j}\right) \in l_{2} \backslash W\right.$ and $\left.\sum_{j=1}^{\infty} x_{j}^{2}=1\right\}$. We observe that $\eta_{2} \sim M_{1} \sim\left(l_{2} \backslash W\right) \sim l_{2}$. The first homeomorphism follows by projection from the point $\left(x_{i}\right)$ for which $x_{1}=-1$ and $x_{j}=0$ for $j>1$. The second homeomorphism follows by the formula $\left(1, x_{2}, x_{3}, \cdots\right) \rightarrow\left(x_{2}, x_{3}, x_{4}, \cdots\right)$ and the third by Statement $(D)$ of $\S 2$. Thus $l_{2}$ is homeomorphic to $l_{2}$.

We note, for use in $\$ 5$, that

$$
\tilde{l}_{2}=\left\{\left(x_{j}\right) \mid\left(x_{j}\right) \in l_{2}, \sum_{j=1}^{\infty} x_{j}^{2}=1 \text { and, for each } i \geqq 1, \sum_{j=1}^{i} x_{j}^{2}<1\right\} \text {. }
$$

5. Description of the homeomorphism $f$ of $\tilde{l}_{2}$ into $s$. With $\left(x_{i}\right)$ denoting a point of $\tilde{l}_{2}$ and $\left(y_{i}\right)$ denoting a point of $s$ (where $0<y_{i}<1$, 
for each $i$ ) we define a function $f$ coordinatewise as:

$$
\begin{aligned}
& y_{1}=\left(1+x_{1}\right) / 2 \text { and for each } i>1, \\
& y_{i}=\frac{\left(1-\sum_{j=1}^{i-1} x_{j}^{2}\right)^{1 / 2}+x_{i}}{2\left(1-\sum_{j=1}^{i-1} x_{j}^{2}\right)^{1 / 2}} .
\end{aligned}
$$

The verification of the following four properties will complete the proof of our Theorem I:

(1) $f$ is one-to-one from $l_{2}$ into $s$,

(2) $f$ is continuous,

(3) $f^{-1}$ is continuous,

(4) $s \backslash f\left(\tilde{l}_{2}\right)$ is the countable union of compact sets.

(1) For any $i>0$ and any point $\left(x_{i}\right) \in \tilde{l}_{2}$, we have $\sum_{j=1}^{i} x_{j}^{2}<1$. Therefore $\left|x_{i}\right|<\left(1-\sum_{j=1}^{i-1} x_{j}^{2}\right)^{1 / 2}$ and thus $0<y_{i}<1$. Hence $f$ carries $\tilde{l}_{2}$ into $s$. Also if $\left(x_{i}\right)$ and $\left(x_{i}^{\prime}\right)$ are different points of $\tilde{l}_{2}$ with images $\left(y_{i}\right)$ and $\left(y_{i}^{\prime}\right)$ in $s$, then there is a least number $k$ such that $x_{k} \neq x_{k}^{\prime}$. From the formulas, $y_{k} \neq y_{k}^{\prime}$ and thus $\left(y_{i}\right) \neq\left(y_{i}^{\prime}\right)$.

(2) Since, by formula, each coordinate function is continuous, then $f$ must be continuous.

(3) To see that $f^{-1}$ is continuous we consider solving for $x_{i}$ in terms of $y_{1}, \cdots, y_{i}$. Clearly, for each $i, x_{i}$ is a continuous function of $y_{1}, \cdots, y_{i}$. Also we know that for $\left(x_{i}\right) \in \tilde{l}_{2}, \sum_{i=1}^{\infty} x_{i}^{2}=1$. Therefore $f^{-1}$ must be continuous since convergence in the norm and convergence in each coordinate gives convergence in $l_{2}$.

(4) We wish to verify that $s \backslash f\left(\tilde{l}_{2}\right)$ is a countable union of compact sets. Since $l_{2}$ is a separable complete metric space, we know that $l_{2}$ and thus $\tilde{l}_{2}$ are absolute $G_{\delta}$ 's. Letting $I^{\infty}=\prod_{i=1}^{\infty} I_{i}$ where $I_{i}$ is the closed interval $[0,1]$, we have $f\left(\tilde{l}_{2}\right) \subset s \subset I^{\infty}$ and we know that $I^{\infty} \backslash f\left(\tilde{l}_{2}\right)$ is a countable union of compact sets $\left(T_{i}\right)_{i \gtrless 1}$.

For each $j>0$, let $R_{j}=\left\{\left(y_{i}\right) \mid\left(y_{i}\right) \in s\right.$ and, for each $i, 1 / 2^{j} \leqq y_{i} \leqq 1$ $\left.-1 / 2^{j}\right\}$. Then $R_{j}$ is a compact subset of $s$. Since $\left\{R_{j} \cap T_{i} \mid i, j>0\right\}$ is a countable union of compact sets, it suffices to show that for any point $\left(y_{i}\right) \in\left(s \backslash \bigcup_{j=1}^{\infty} R_{j}\right)$, there is a point $\left(x_{i}\right) \in \tilde{l}_{2}$ for which $f\left(\left(x_{i}\right)\right)$ $=\left(y_{i}\right)$.

Clearly, for each point $\left(y_{i}\right) \in s$, the formulas solved for the $y_{i}$ 's yield a particular sequence $\left(x_{i}\right)$ with $\sum_{i=1}^{\infty} x_{i}^{2} \leqq 1$. We note that $\left(x_{i}\right)$ may have only finitely many nonzero coordinates but, in this case, $\sum_{i=1}^{\infty} x_{i}^{2}<1$. 
Given $\left(y_{i}\right) \in\left(s \backslash \bigcup_{i=1}^{\infty} R_{j}\right)$ and any $\delta>0$ there exist an integer $k$ and positive numbers $\epsilon_{1}$ and $\epsilon_{2}$ such that

$$
0<y_{k}<\epsilon_{1} \text { or } 1-\epsilon_{1}<y_{k}<1,
$$

and this statement implies

$$
\left(1-\sum_{j=1}^{k-1} x_{j}^{2}\right)^{1 / 2}-\epsilon_{2}<\left|x_{k}\right|<\left(1-\sum_{j=1}^{k-1} x_{j}^{2}\right)^{1 / 2},
$$

and this statement implies

$$
0<\left(1-\sum_{j=1}^{k} x_{j}^{2}\right)<\delta \text { and } x_{k} \neq 0 .
$$

Therefore there is a point $\left(x_{i}\right)$ of $\tilde{l}_{2}$ such that $f\left(\left(x_{i}\right)\right)=y_{i}$.

\section{BIBLIOGRAPHY}

1. R. D. Anderson, Topological properties of the Hilbert Cube and the infinite product of open intervals, Trans. Amer. Math. Soc. (to appear).

2. S. Banach, Théorie des opérations linéaires, Monografie Matematyczne, Warsaw, 1932.

3. C. Bessaga, On topological classification of complete linear metric spaces, Fund. Math. 55 (1965), 251-288.

4. C. Bessaga and A. Pełczynski, Some remarks on homeomorphisms of F-spaces, Gauthier-Villars, Bull. Acad. Polon. Sci. Ser. Sci. Math. Astr. Phys. 10 (1962), 265270.

5. M. Fréchet, Les espaces abstraits, Paris, 1928.

6. M. I. Kadec, On topological equivalence of separable Banach spaces, Dokl. Akad. Nauk. SSSR (to appear).

Louisiana State University, Baton Rouge 